\title{
Diurnal and Nocturnal Behaviour of Airborne Cryptomeria japonica Pollen Grains and the Allergenic Species in Urban Atmosphere of Saitama, Japan
}

\author{
Qingyue Wang*, Shinichi Nakamura, Senlin Lu ${ }^{1)}$, Daisuke Nakajima ${ }^{2)}$, Miho suzuki, \\ Kazuhiko Sekiguchi and Makoto Miwa ${ }^{3)}$ \\ Graduate School of Science and Engineering, Saitama University, Shimo-Okubo 255, Sakura, Saitama, Saitama 338-8570, Japan \\ ${ }^{1)}$ School of Environmental and Chemical Engineering, Shanghai University, 99, Shangda Road, Baoshan, Shanghai 200444, China \\ ${ }^{2}$ Resarch Center for Environmental Risk, National institute for environmental studies, Onogawa 16-2, Tsukuba, Ibaraki 305-8506, Japan \\ ${ }^{3)}$ Center for Environmental Science in Saitama, Kamitanadare 914, Kisai, Kitasaitama, Saitama 347-0115, Japan
}

*Corresponding author. Tel: +81-48-858-3733, E-mail: seiyo@mail.saitama-u.ac.jp

\begin{abstract}
Japanese cedar (Cryptomeria japonica) pollinosis is the most popular pollinosis in Japan. It has been reported that Cryptomeria japonica pollen allergenic species are suspended as fine particles in the urban atmosphere. These allergenic fine particles are responsible for inducing asthma by breaking into the lower respiratory tract. It has also been found that pollinosis symptoms on the sufferers appear mainly at night-time by the results from epidemiological studies. However, the exact reason for these phenomena is not yet clarified. In this study, the diurnal and nocturnal behaviours of Cryptomeria japonica pollen grains and their allergenic species in the urban area of Saitama city of Kanto Plain were investigated. Airborne pollen grains and allergenic Cry $\mathrm{j} 1$ concentrations in total suspended particulate matter (TSP) were investigated at two sampling sites, a heavy traffic road (roadside site) and at the balcony of the $10^{\text {th }}$ floor of the Building of Research and Project of Saitama University (general urban site). The latter sampling site where located about $300 \mathrm{~m}$ away from the roadside site was used as a general urban site unaffected by automobile traffic. The airborne pollen counts were measured with a real-time pollen monitor. Cry $\mathrm{j} 1$ particles were collected with two high volume air samplers, and these concentrations were measured by surface plasmon resonance method with a Biacore J system. The diurnal variation of the airborne pollen counts was similar to the trends of temperature and wind speed during the day-time; whereas its tendency with wind speed trend was not observed during the night-time. Airborne pollen counts were lower with northern wind than with southern wind because the pollen comes from the mountainous areas, and the mountains in the south
\end{abstract}

are closer, about half the distance to the northern mountains. It is suggested that the peaks of airborne pollen counts during night-time in the sampling site occurred by transport of pollen grains released during day-time in the mountainous forest areas, located c.a. $100 \mathrm{~km}$ away from the sampling site. On the roadside site the allergenic Cry $\mathrm{j} 1$ concentrations were higher than at the general urban site, nevertheless pollen grains counts were lower. These results suggested that worsening of pollinosis symptoms during nighttime in urban area was caused by transport of pollen grains during day-time in the mountainous forest areas. Moreover, pollen allergenic species become different morphology from pollen grain at roadside site, and the subsequent pollen grains re-suspension by automobile traffic.

Key words: Cryptomeria japonica pollen, Diurnal behaviour, Allergenic Cry j 1, Suspended particulate matters, Urban atmosphere

\section{INTRODUCTION}

Japanese cedar (Cryptomeria japonica) trees had been afforested in mountainous areas from mid-1940s to 1950 s for post-war lumber demand in Japan (Okuda et al., 2006). Currently, these trees have grown up over 30 years and heavily release pollen grains (Kitamura et al., 2005). The prevalence of Japanese cedar pollinosis had become over $26.5 \%$ in Japan, especially it had become higher than $30 \%$ in the urban areas in Kanto (Murayama et al., 2010). The forest cannot be seen in urban areas of Kanto Plain, Japan. The airborne Cryptomeria japonica pollen grains in the urban areas are transported from the mountainous forest areas, several hundred kilometres away (Kanazashi and Suzuki, 2010). Moreover, since pollinosis symptoms on the 
sufferers are reported mainly at night-time (Maeda et al., 2008), it suggests that airborne pollen grains and allergenic species may be higher during night-time.

Cryptomeria japonica pollen has two main allergenic species, so-called Cry j 1 and Cry j 2, which are responsible for pollinosis. These allergenic species are located in the pollen wall and ubisch bodies on pollen surface and starch granules in the pollen grains, Cry j 1 and Cry j 2 respectively (Nakamura et al., 2004). Because of the high prevalence of pollinosis in urban areas, air pollutants and automobile traffics are considered to produce synergetic effects on the allergens (Karle et al., 2012; Rogerieux et al., 2007; Behrendt et al., 1997). Especially, symptoms of asthma on patients induced by Cryptomeria japonica pollen allergenic species have been observed (Maeda et al., 2008), and worsening during pollen scattering season (Taylor and Jonsson, 2004). It is suggested that this asthma is caused by the breaking of small-sized Cryptomeria japonica pollen allergenic species into lower respiratory tract. In contrast particle size of the pollen grains is c.a. $30 \mu \mathrm{m}$, it had been reported that small-sized Cry $\mathrm{j} 1$ species were suspended as fine particles which particle size were below $1.1 \mu \mathrm{m}$ in urban atmosphere (Wang et al., 2008). It is also thought that particles containing small-sized allergenic species and pollen grains deposited onto the paved asphalt road materials are re-suspended by automobile traffic.

The behaviour of Cryptomeria japonica pollen grains and of its allergenic species in the urban atmosphere has been studied in a few reports. It has also been found that pollinosis symptoms on the sufferers appear mainly at night-time by the results from epidemiological studies. However, actual cause of these phenomena is not yet clarified. Moreover, since allergenic particle were collected over several days in these reports, diurnal behaviour of these allergenic particles was also unknown. In this study, the diurnal behaviours of Cryptomeria japonica pollen grains and their allergenic species in the urban of Saitama city of Kanto Plain, Japan were investigated.

\section{MATERIALS AND METHODS}

\section{1 Location of Sampling Site}

Monitoring of airborne pollen counts and the collection of particles containing allergenic Cry $\mathrm{j} 1$ were performed in Saitama University, where is located in the urban area of Kanto Plain, Japan $\left(35.86^{\circ} \mathrm{N}\right.$ and 139.61 ${ }^{\circ}$ E) (site IV in Fig. 1(a)). The forests of Cryptomeria japonica are around mountainous areas of Chichibu and neighbour prefectures, several hundred kilometres away from the sampling site. Most Cryptomeria japonica pollen grains in the sampling site were transported from those circumjacent mountainous areas. The others observation sites in Fig. 1(a) observe pollen counts as part of monitoring network system of the Ministry of the Environment (http://kafun.taiki.go.jp/; 22 Oct. 2012).
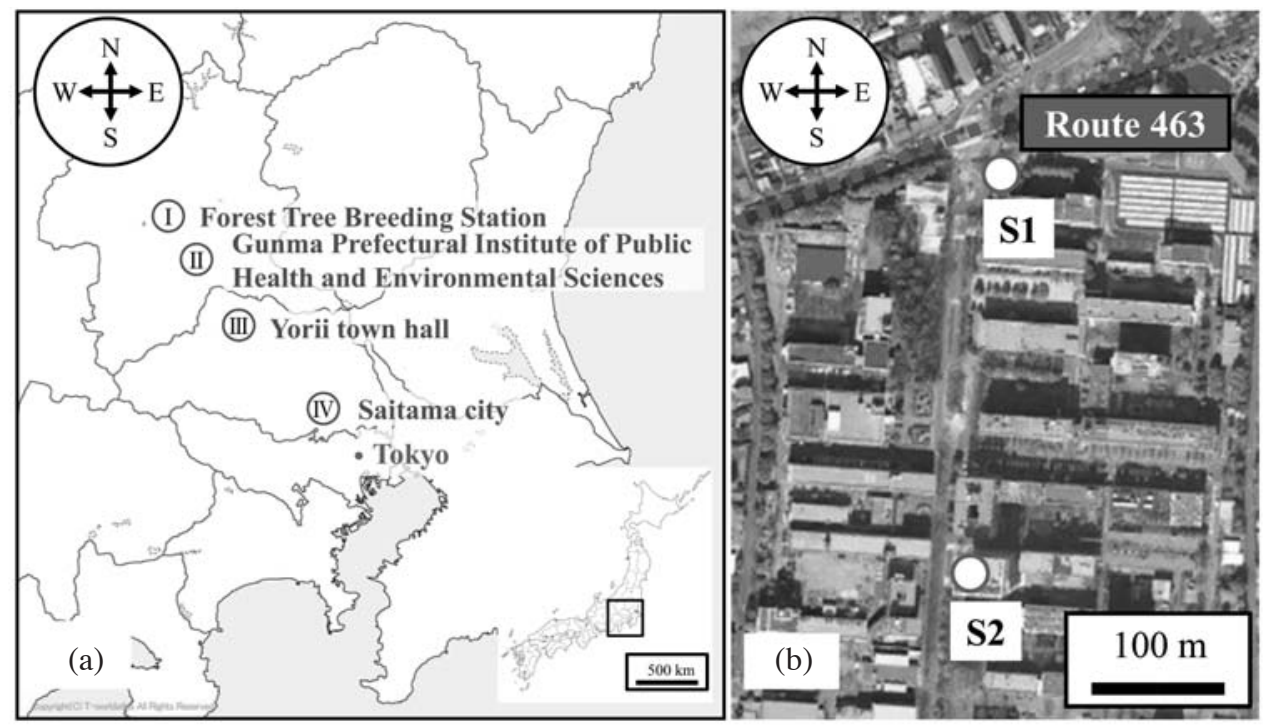

Fig. 1. Sampling location. (a) Site I: Forest Tree Breeding Station, Gunma; Site II: Gunma Prefectural Institute of Public Health and Environmental Sciences, Gunma; Site III: Yorii town hall, Saitama and Site IV: Saitama city hall and Saitama University, Saitama, Japan. (b) The sampling sites in Saitama University (S1: 2 m near roadside, S2: 300 m away from the roadside, the red dashed line indicates National road 463). 
The diurnal air samples were taken at two sampling sites in Saitama University (Fig. 1(b)). A roadside site (S1) where is located $2 \mathrm{~m}$ away from the vehicles pass in the National Route 463, in Saitama University. Another sampling site is settled in the balcony of the $10^{\text {th }}$ floor of the Building of Research and Project of Saitama University (S2) (here after described as "general urban site"), about $300 \mathrm{~m}$ away from the roadside site (S1), as less effect site of automobile traffic. The diurnal air samples were collected during 12-hours sampling period (the day-time; 8:00-20:00 and the night-time; 20:00-8:00) from 26 March to 8 April, 2011.

\subsection{Monitoring of Diurnal Airborne Pollen Counts}

The diurnal airborne pollen counts were monitored and calculated with a real-time pollen monitor (Model KH3000-01, Yamatronics Co., Ltd., Japan) operated at a flow rate $4.1 \mathrm{~L} / \mathrm{min}$. The pollen monitor counts particles which have characteristics of previously memorized optical scatter pattern of 28 to $35 \mu \mathrm{m}$ of diameter and spherical shape, such as the pollen grains, using optical system by forward and side scatter with a laser diode (Seno et al., 2002). The pollen counts of monitor are well correlated with those of the Burkard pollen sampler which is used as the standard pollen sampler in European countries (Takahashi et al., 2001a). The results of airborne pollen counts were converted into 12-hours average counts of the pollen per $1.0 \mathrm{~m}^{3}$ volume (counts $/ \mathrm{m}^{3}$ ).

\section{3 Investigation of Allergenic Cry j 1 Concentrations in Airborne Particles}

High-volume air samplers (Model HV-1000R, Shibata Scientific Technology Co., Ltd., Japan) were used to collect atmospheric particles. Total suspended particulate matter (TSP) including Cryptomeria japonica pollen grains and their allergenic fine particles were collected on quartz fibre filters (Tokyo Dylec Co., Ltd., Japan).

Allergenic Cry $\mathrm{j} 1$ species were extracted using the following method: A circular area with a diameter of $47 \mathrm{~mm}$ was cut from the quartz fibre filters, this area was added $2 \mathrm{~mL}$ of Cry $\mathrm{j} 1$ extraction solution $(150 \mathrm{mM}$ of $\mathrm{NaCl}, 0.125 \mathrm{M}$ of $\mathrm{NH}_{4} \mathrm{CO}_{3}, 3 \mathrm{mM}$ of EDTA, 0.005 wt $\%$ of Tween 20 and $10 \mathrm{mM}$ of HEPES buffer solution) (Wang et al., 2010) and left overnight at $4^{\circ} \mathrm{C}$, and then shook at $192 \mathrm{rpm}$ for 1 hour. Afterwards the samples were centrifuged for $30 \mathrm{~min}$ at $6,000 \mathrm{rpm}$, and the supernatant solutions were picked up. Finally, allergenic Cry $\mathrm{j} 1$ concentrations on these supernatants were measured by a Biacore J system (GE Healthcare Co., Ltd., Japan) based on the surface plasmon resonance
(SPR) method.

The SPR method was used to detect Cry $\mathrm{j} 1$ concentrations by using allergen-specific monoclonal antibodies and the Biacore $\mathbf{J}$ system, as previously described (Wang et al., 2012; Takahashi et al., 2001b) with the following minor modifications: Allergenic Cry $\mathrm{j} 1$ concentrations in measured samples were determined based on Cry j 1 monoclonal antibody (MAb) (clone 013, Asahi Food \& Healthcare Co., Ltd., Japan) immobilized on a CM5 sensor chip (GE Healthcare Co., Ltd., Japan) by using the SPR method with the Biacore $\mathrm{J}$ system. Approximately $700 \mu \mathrm{g}$ of Cry j 1 MAb was immobilized on the chip. The regeneration and dissociation of antigen-antibody complex reaction on the CM5 sensor chip were performed by $70 \mu \mathrm{L}$ of $\mathrm{pH} 2.0$ glycine-HCl (GE Healthcare Co., Ltd., Japan). Finally, the results were converted into Cry $\mathrm{j} 1$ concentration $\left(\mathrm{ng} / \mathrm{m}^{3}\right)$ in the urban atmosphere (Wang et al., 2012).

\section{4 Meteorological Data during the Sampling Periods}

Meteorological data were supplied from the Saitama Institute of Public Health and the Okubo Purification Plant which only located around 700 meters from our air sampling sites. Data on precipitation, wind speed, wind direction and temperature were acquired.

\section{RESULTS AND DISCUSSION}

\subsection{Diurnal and Nocturnal Behaviour of the Airborne Pollen Grains}

Fig. 2 indicates the diurnal and nocturnal variations of meteorological data (temperature, precipitation (Fig. 2(a)), wind speeds and wind direction (Fig. 2(b)) and the airborne pollen counts and allergenic Cry $\mathrm{j} 1$ concentrations at the roadside site and the general urban site from 26 March to April 8 in 2011 (Fig. 2(c)). In both sites, the highest airborne pollen counts were 764 counts $/ \mathrm{m}^{3}$, and average pollen counts were 180 counts $/ \mathrm{m}^{3}$. The highest airborne Cry j 1 concentrations were $50 \mathrm{ng} / \mathrm{m}^{3}$, and average Cry j 1 concentrations were $18 \mathrm{ng} / \mathrm{m}^{3}$ during the observation period. It has been reported that the symptoms of pollinosis appear when reaching a few $\mathrm{pg} / \mathrm{m}^{3}$ of allergenic Cry j 1 concentrations in the atmosphere (Takahashi et al., 2001b). Thus, the airborne Cry $\mathrm{j} 1$ particles suspended above the threshold level that triggers pollinosis symptoms during the observation.

The diurnal variation of the airborne pollen counts showed good correlation with temperature, and this is in good agreement with the report by other researchers (Bartkova-Scevkova, 2003). At the latter air sampling period, the airborne pollen grain counts notably incr- 

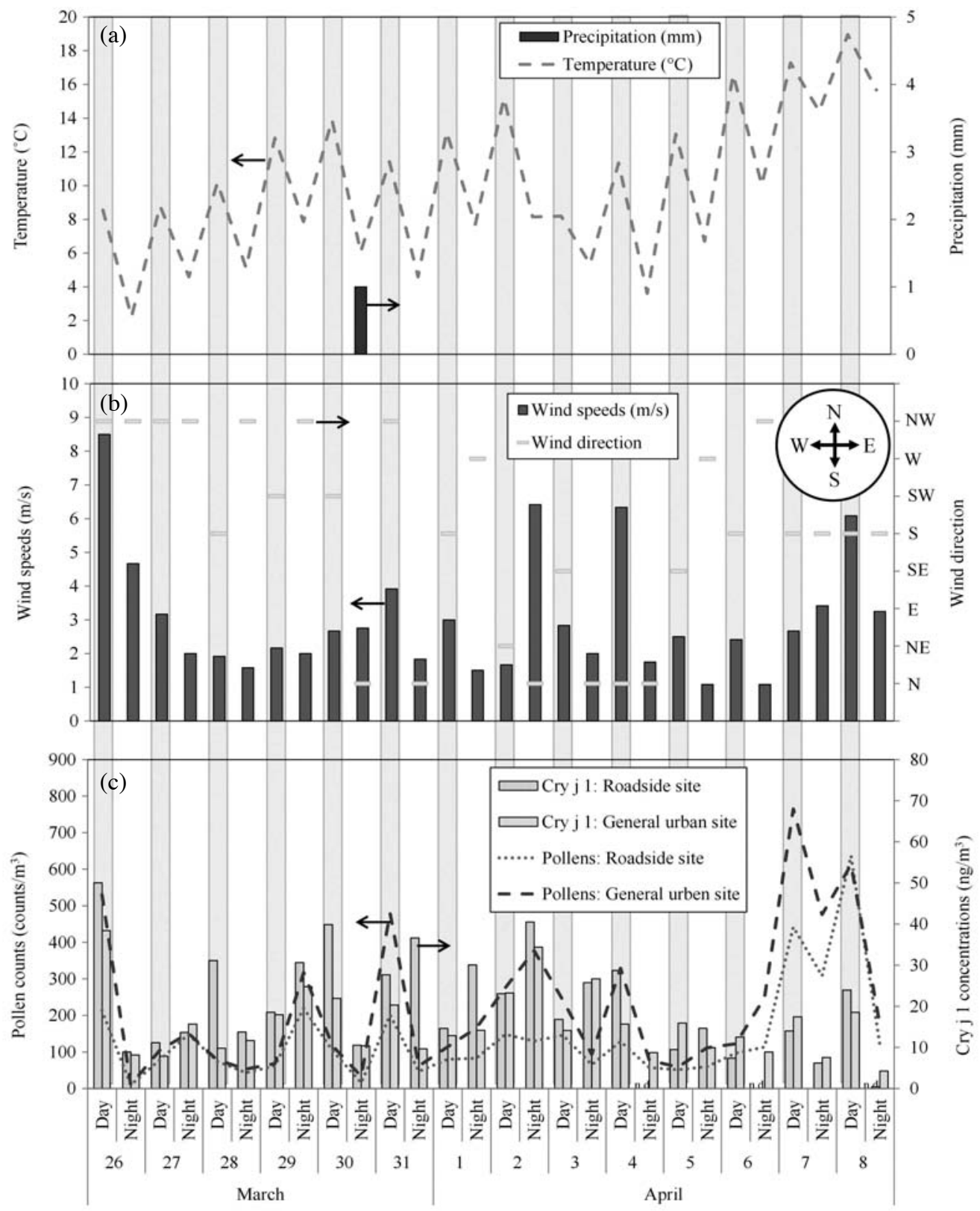

Fig. 2. Diurnal variation of temperature, precipitation (a), wind speed, wind direction (b), pollen counts and allergenic Cry $\mathrm{j} 1$ concentrations (c) at the roadside site and the general urban site. Meteorological data were provided by the Saitama Institute of Public Health and the Okubo Purification Plant.

eased with increasing temperature. It has reported that pollinosis suffers are induced symptoms by temperature above $15^{\circ} \mathrm{C}$ (Dejima et al., 1992). Therefore, it is thought that the airborne pollen grains are also increased by high temperature.

The trend of the airborne pollen grains was similar to the trend of wind speed during day-time; whereas this similarity was not observed during night-time. It is thought that the airborne pollen counts were related to wind speed, because under stronger winds more pollen grains are released from the source trees. During night-time, it was also thought that only a few pollen grains are released from the original trees of emission sources, since male flowers which contain pollen grains close under dark conditions.

During night-time in 30 March, the airborne pollen grains and Cry $\mathrm{j} 1$ concentrations decreased after $1 \mathrm{~mm}$ of rain precipitation. Airborne particles disappeared 
Table 1. Hourly data of wind direction during night-time of 2 Apr. and day-time of 7 Apr. at Saitama, Saitama.

\begin{tabular}{|c|c|c|c|}
\hline \multicolumn{2}{|c|}{ At night-time of 2 Apr. } & \multicolumn{2}{|c|}{ At day-time of 7 Apr. } \\
\hline $\begin{array}{c}\text { Observation } \\
\text { time }\end{array}$ & $\begin{array}{c}\text { Wind } \\
\text { direction }\end{array}$ & $\begin{array}{l}\text { Observation } \\
\text { time }\end{array}$ & $\begin{array}{l}\text { Wind } \\
\text { direction }\end{array}$ \\
\hline 20 & NNW & 8 & WNW \\
\hline 21 & $\mathrm{~N}$ & 9 & $\mathrm{~N}$ \\
\hline 22 & NNW & 10 & NNE \\
\hline 23 & NNW & 11 & SW \\
\hline 24 & NNW & 12 & SSW \\
\hline 1 & NNW & 13 & $\mathrm{~S}$ \\
\hline 2 & NNW & 14 & SSE \\
\hline 3 & NNW & 15 & $\mathrm{~S}$ \\
\hline 4 & NNW & 16 & $\mathrm{~S}$ \\
\hline 5 & NNW & 17 & $S$ \\
\hline 6 & NW & 18 & $\mathrm{~S}$ \\
\hline 7 & NNW & 19 & SSW \\
\hline 8 & NNW & 20 & $\mathrm{~S}$ \\
\hline
\end{tabular}

from the atmosphere by trapping into rainwater, due to the so-called wash-out phenomenon. The airborne pollen grains and particles containing allergenic Cry $\mathrm{j}$ 1 also disappeared by trapping into rainwater.

Airborne pollen counts were lower with northern wind than with southern wind. The mountains in the south are closer, about half the distance to the northern mountains. Temperature also increased with southern wind. It is thought that pollen counts depend more on temperature rather than on wind speed. On the other hand, pollen counts are strongly affected by wind speed when northern wind blows, because of the longer transportation distance.

The airborne pollen counts peaks were observed when winds blew mostly from northwest and southwest. During the night-time of 2 April, wind direction in sampling sites was kept northwest (Table 1). Observation sites of Maebashi and Yorii, near the site II and site III in Fig. 1, where windward of sampling sites were also kept northwest wind (Data not shown). Thus, it was estimated that the airborne pollen grains were transported from mountainous areas of Gunma prefecture located c.a. $100 \mathrm{~km}$ away from the sampling site. At day-time of 7 April, winds blew from northwest till 10 a.m., subsequently southern wind blew till nighttime. Thus, it was estimated that the airborne pollen grains were mostly transported from mountainous areas to the west of Tokyo metropolitan area and located c.a. $50 \mathrm{~km}$ away from the sampling site.

\section{2 Airborne Pollen Counts Peaks during the Night-time in Urban Areas of Kanto Plain}

Fig. 3 shows the tracking data of the airborne pollen peaks at night-time of 2 April using monitoring network

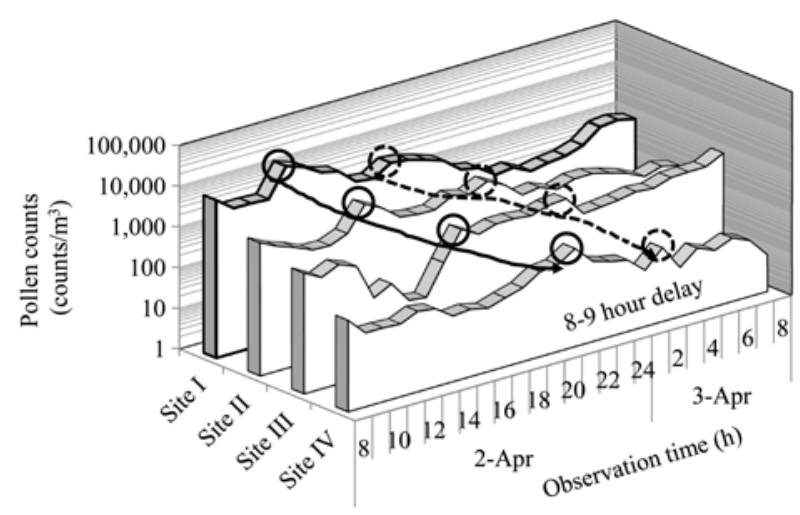

Fig. 3. Time variation of airborne pollen counts at 2 April to 3 April in 2011. About 8 to 9 hour peak delays of airborne pollen counts were observed at site IV of the urban compared with the site I of original emission sources. Here, site I: Forest Tree Breeding Station, Gunma; site II: Gunma Prefectural Institute of Public Health and Environmental Sciences, Gunma; site III: Yorii town hall and site IV: Saitama city hall, Japan; Data were downloaded from the Ministry the of Environment (http://kafun.taiki.go.jp/; 22 Oct. 2012).

system of the Ministry of the Environment (http:// kafun.taiki.go.jp/; 22 Oct. 2012). Observation sites I, II, III and IV are related to each observation site of Fig. 1(a).

The Forest Tree Breeding Station as the observation site I is located nearest mountainous area of original emission sources of pollen grains. The pollen peak appeared at site I at the noon on 2 April were moving to the southern observation site with decreasing pollen counts by diffusion, since wind blew from north at night-time of 2 April. The pollen peak went through to site II at 14:00, site III at 17:00 and site IV, where located near our sampling sites, at 21:00 on 2 April.

Another pollen peak appeared in site I at 18:00 also went through site IV at 2:00 on 3 April. The airborne pollen grains arriving to our urban sampling site need 8 to 9 hours, which is about 8 to 9 hour delay shown in Fig. 3 after being released from the Japanese cedar (Cryptomeria japonica) forest as the original emission sources. Thus, the pollen grains from original emission trees in the forest in the mountainous areas, released during the day-time, were observed at our urban sampling site during the night-time. The transportation times were consistent with the average wind speed, 2 $\mathrm{m} / \mathrm{s}$ at the day-time of 2 April. As the result, it was thought that appearance of peaks of the airborne pollen counts during the night-time in urban area of Kanto Plain was produced by transportation of pollen grains released from trees in mountainous areas during the day-time. 

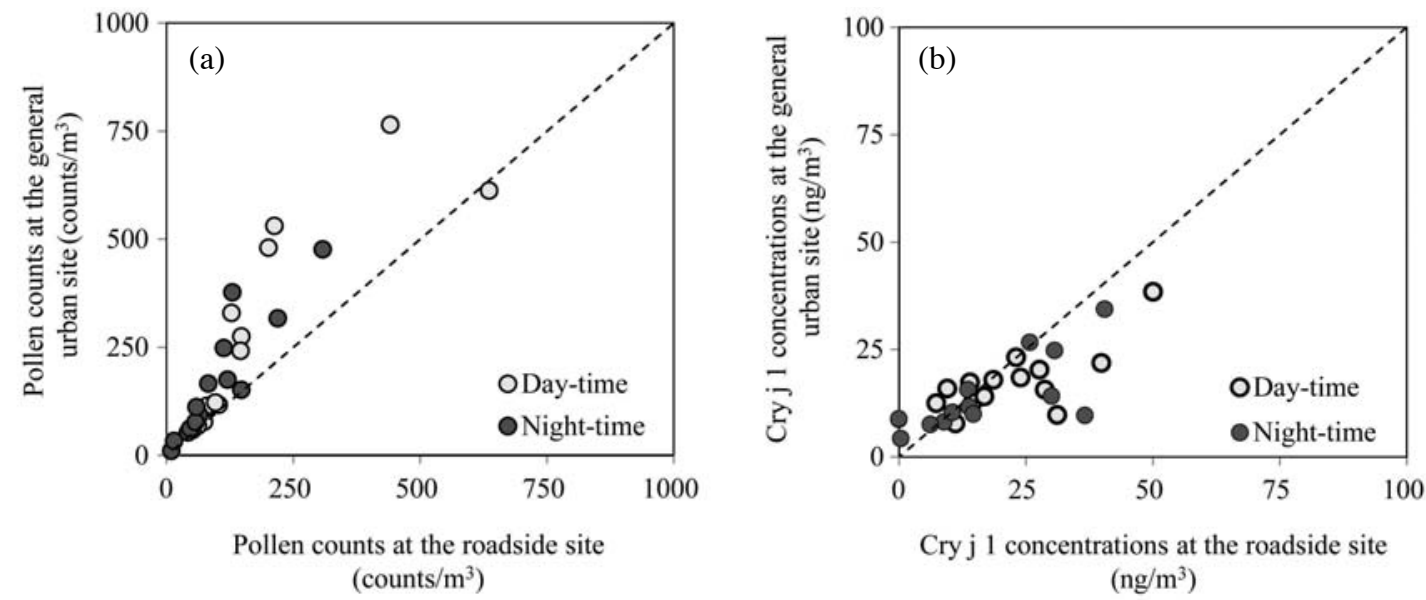

Fig. 4. Correlation between the roadside site and the general urban site in (a) pollen counts and (b) allergenic Cry j 1 concentrations.

\subsection{Comparison between the Airborne Pollen Counts and Allergenic Cry j 1 Concentrations}

The correlations about pollen counts and allergenic Cry $\mathrm{j} 1$ concentrations between the roadside site and the general urban site are shown in Fig. 4(a) and Fig. 4(b) respectively. Cry j 1 concentrations between the roadside site and the general urban site indicated similar behaviour in common with pollen counts. Cry $\mathrm{j} 1$ concentrations at the roadside site were higher than at the general urban site; whereas pollen counts were lower. Differences of these behaviours between daytime and night-time were not observed.

It is thought that the airborne pollen grains in the sky of urban areas keep their morphology, since these airborne pollen grains are just transported from releasing forest sources in mountainous areas, and not affected by air pollutants and automobile traffic. In the urban atmosphere, the airborne pollen grains underwent morphological change such as burst of pollen grains and adhesion of other suspended particulate matter (Okuyama et al., 2007), and small-sizing of particles containing allergenic Cry j 1 (Wang et al., 2008). We presume that pollen grains were more suspended on the roadside site, since there were more pollen grains transported and re-suspended. However, the results were different from our thinking. For the results, it is suggested that morphological change of pollen grains including small-sizing is enhanced at roadside by heavy traffic.

It may be thought that during the deposition process of transported pollen grains on the asphalt, their counts would decrease by deposition on trees. However, the effect of dry deposition was low, since Cry $\mathrm{j} 1$ concentrations on the roadside site were higher than ones at the general urban site. The results suggested that the airborne pollen grains in the urban atmosphere include morphological changed pollen grains were uncounted by the pollen monitor device using optical systems such as the monitor of KH-3000-01. This device is widely used at the monitoring network system of the Ministry of the Environment, Japan (http://kafun.taiki. go.jp/; 22 Oct. 2012). It is possible that the real airborne pollen counts in urban atmosphere are underestimated and this may lead to serious damages on pollinosis sufferers.

\section{CONCLUSIONS}

In this study, it was investigated the diurnal and nocturnal behaviour of Cryptomeria japonica pollen grains and its allergenic species in urban area in Kanto Plain, Japan. Aims of this study were to gain knowledge about the causation of worsening of pollinosis symptoms during night-time and formation of fine particles containing allergenic Cry $\mathrm{j} 1$ in urban area. Peaks of pollen grains were observed during night-time in the sampling sites. Since the pollen counts were higher than during the day-time in the peaks, it is suggested that appearances of the pollen peaks during night-time in urban area are caused by transport and deposition of pollen grains. Allergenic Cry $\mathrm{j} 1$ concentrations at roadside site were higher than ones at the general urban site, nevertheless pollen grains counts were lower at roadside site. These results suggested that worsening of pollinosis symptoms during night-time in urban area was caused by the transport of pollen grains during daytime from the original forest of mountainous areas. Moreover, pollen allergenic species become different morphology from pollen grain at roadside site, and the subsequent pollen grains re-suspension by heavy 
automobile transportation.

\section{ACKNOWLEDGEMENT}

Some works of this study were supported by the Special Funds for Innovative Area Research (No. 20120015, FY2008 FY2012) and Grant-in-Aid for Challenging Exploratory Research (No. 23651 005, FY2011 FY2012) of Japanese Ministry of Education, Culture, Sports, Science and Technology (MEXT), Japan.

\section{REFERENCES}

Bartkova-Scevkova, J. (2003) The Influence of Temperature, Relative Humidity and Rainfall on the Occurrence of Pollen Allergens (Betula, Poaceae, Ambrosia artemisiifolia) in the Atmosphere of Bratislava (Slovakia). International Journal of Biometeorology 48(1), 1-5.

Behrendt, H., Becker, W.M., Fritzsche, C., Sliwa-Tomczok, W., Tomczok, J., Friedrichs, K.H., Ring, J. (1997) Air Pollution and Allergy: Experimental Studies on Modulation of Allergen Release from Pollen by Air Pollutants. International Archives of Allergy and Immunology 113, 69-74.

Dejima, K., Takenaka, H., Mizukoshi, H., Saito, K., Kawata, R., Takagi, N., Saito, Y., Shoji, H. (1992) A Relationship between the Annual Onset Day of Japanese Cedar Pollinosis and Pollen Dispersion. The Japanese Society of Allergology 41(9), 1405-1412 (in Japanese).

Kanazashi, T., Suzuki, M. (2010) The Estimation of the Pollen Source Areas of Cryptomeria japonica D. Don which Have High Contributions to the Japanese Metropolitan Region. The Japanese Forest Society 92, 298303 (in Japanese).

Karle, A.C., Oostingh, G.J., Mutschlechner, S., Ferreira, F., Lackner, P., Bohle, B., Fischer, G.F., Vogt, A.B., Duschl, A. (2012) Nitration of the Pollen Allergen Bet v 1.0101 Enhances the Presentation of Bet v 1-Derived Peptides by HLA-DR on Human Dendritic cells. Plos One 7(2), e31483.

Kitamura, T., Shiomori, T., Fujimura, T., Suzuki, H. (2005) Allergy (1) Nasal Allergy by Pollen. Journal of Aerosol Research 20(1), 54-57 (in Japanese).

Maeda, Y., Akiyama, K., Shida, T. (2008) A Clinical Study of Japanese Cedar (Cryptmeria japonica) Pollen-Induced Asthma. Allergology International 57, 413-417.

Murayama, K., Baba, K., Okubo, K. (2010) Regional Differences in the Prevalence of Japanese Cedar-Pollen Allergy. The Japanese Society of Allergology 59(1), 47-54 (in Japanese).
Nakamura, S., Sato, F., Nakamura, N. (2004) Immunocytochemical Localization of Cry j 1 and Cry j 2-the Allergenic Proteins of Japanese Cedar Pollen-in the Germinated Pollen. Japanese Journal of Palynology 50(1), 15-22 (in Japanese).

Okuda, M., Usami, A., Kisikawa, R. (2006) Study of Recent Yearly Trend of Increase in Airborne Pollen of Japanese Cedar and Cypress Pollen. The Japanese Society of Allergology 55(12), 1531-1535 (in Japanese).

Okuyama, Y., Matsumoto, K., Okochi, H., Igawa, M. (2007) Adsorption of Air Pollutants on the Grain Surface of Japanese Cedar Pollen. Atmospheric Environment 41, 253-260.

Rogerieux, F., Godfrin, D., Sènèchal, H., Motta, A.C., Marlière, M., Peltre, G., Lacroix, G. (2007) Modifications of Phleum pretense Grass Pollen Allergens following Artificial Exposure to Gaseous Air Pollutants $\left(\mathrm{O}_{3}\right.$, $\mathrm{NO}_{2}, \mathrm{SO}_{2}$ ). International Archives of Allergy and Immunology 143, 127-134.

Seno, S., Dake, Y., Sakoda, T., Saito, Y., Ikeda, H., Kitano, H., Kitajima, K., Enomoto, T. (2002) Survey of Pollen in Wakayama City with Real-Time Pollen Counter. Nihon Jibiinkoka Gakkai Kaiho 105(3), 232-239 (in Japanese).

Takahashi, Y., Kawashima, S., Fujita, T., Ito, C., Togashi, R., Takeda, H. (2001a) Comparison between Real-Time Pollen Monitor KH-3000 and Burkard Sampler. Japanese Journal of Allergology 50(12), 1136-1142 (in Japanese).

Takahashi, Y., Ohashi, T., Nagoya, T., Sakaguchi, M., Yasueda, H. (2001b) Possibility of Real-Time Measurement of an Airborne Cryptomeria japonica Pollen Allergen Based on the Principle of Surface Plasmon Resonance. Aerobiologia 17, 313-318.

Taylor, P.E., Jonsson, H. (2004) Thunderstorm Asthma. Current Allergy and Asthma Reports 4, 409-413.

The Ministry of the Environment (the Monitoring Network System), last access: 22 Oct. 2012. http://kafun.taiki.go. $\mathrm{jp} /$ (in Japanese).

Wang, Q., Kurihara, K., Kiryu, H., Sakamoto, K., Miwa, M., Uchiyama, I. (2008) Daily Variation Differences between Airborne Japanese Cedar Pollen and its Respirable Allergen Particulate Matters during the Most Severe Pollination Seasons. Earozoru Kenkyu 23, 120126 (in Japanese).

Wang, Q., Nakamura, S., Lu, S., Nakajima, D., Suzuki, M., Sakamoto, K., Miwa, M. (2012) Release Behavior of Small Sized Daughter Allergens from Cryptomeria japonica Pollen Grains during Urban Rainfall Event. Aerobiologia 28, 71-81.

(Received 10 January 2013, revised 21 March 2013, accepted 25 March 2013) 